\title{
Classical eyeblink conditioning using electrical stimulation of caudal MPFC as conditioned stimulus is dependent on cerebellar interpositus nucleus in guinea pigs
}

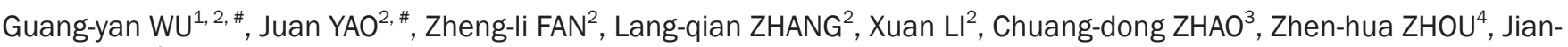 \\ feng SUI ${ }^{1,2, *}$ \\ ${ }^{1}$ Department of Physiology, College of Basic Medical Sciences, Third Military Medical University, Chongqing 400038, China; ${ }^{2}$ Expe- \\ rimental Center of Basic Medicine, College of Basic Medical Sciences, Third Military Medical University, Chongqing 400038, China; \\ ${ }^{3}$ Department of Neurosurgery, General Hospital of Ji-nan Command, Ji-nan 250003, China; ${ }^{4}$ Department of Neurology, Southwest Hos- \\ pital, Third Military Medical University, Chongqing 400038, China
}

\begin{abstract}
Aim: To determine whether electrical stimulation of caudal medial prefrontal cortex (MPFC) as conditioned stimulus (CS) paired with airpuff unconditioned stimulus (US) was sufficient for establishing eyeblink conditioning in guinea pigs, and whether it was dependent on cerebellar interpositus nucleus.

Methods: Thirty adult guinea pigs were divided into 3 conditioned groups, and trained on the delay eyeblink conditioning, short-trace eyeblink conditioning, and long-trace eyeblink conditioning paradigms, respectively, in which electrical stimulation of the right caudal mPFC was used as CS and paired with corneal airpuff US. A pseudo conditioned group of another 10 adult guinea pigs was given unpaired caudal mPFC electrical stimulation and the US. Muscimol ( $1 \mu \mathrm{g}$ in $1 \mu \mathrm{L}$ saline $)$ and saline $(1 \mu \mathrm{L})$ were infused into the cerebellar interpositus nucleus of the animals through the infusion cannula on d 11 and 12, respectively.

Results: The 3 eyeblink conditioning paradigms have been successfully established in guinea pigs. The animals acquired the delay and short-trace conditioned responses more rapidly than long-trace conditioned responses. Muscimol infusion into the cerebellar interpositus nucleus markedly impaired the expression of the 3 eyeblink conditioned responses.

Conclusion: Electrical stimulation of caudal mPFC is effective CS for establishing eyeblink conditioning in guinea pigs, and it is dependent on the cerebellar interpositus nucleus.
\end{abstract}

Keywords: associative learning; memory; eyeblink conditioning; medial prefrontal cortex; cerebellar interpositus nucleus; muscimol; guinea pig

Acta Pharmacologica Sinica (2012) 33: 717-727; doi: 10.1038/aps.2012.32; published online 7 May 2012

\section{Introduction}

Classical eyeblink conditioning is one of the most widely used model systems for studying the behavioral and neurobiological mechanisms of associative learning and memory ${ }^{[1-3]}$. All variant of eyeblink conditioning involve paired presentations of a behaviorally neutral conditioned stimulus (CS; eg, a tone or light) and an unconditioned stimulus (US; eg, a corneal airpuff or periorbital shock). Initially, the organisms could produce only a reflexive eyeblink unconditioned response

\footnotetext{
\#The two authors contributed equally to this work.

* To whom correspondence should be addressed.

E-mail jfsui2003@yahoo.com.cn

Received 2012-02-19 Accepted 2012-03-14
}

(UR) to the US. After hundreds of paired presentations of the CS and the US, the organisms could learn to close the eyes in response to the CS before the onset of the US (called the conditioned response, $\mathrm{CR}$ ). According to the temporal relationship between the CS and the US, there are two commonly used procedures in eyeblink conditioning: trace and delay paradigms. In the trace eyeblink conditioning (TEC), a temporal gap occurs between the offset of the CS and the onset of the US, which is in contrast to the delay eyeblink conditioning (DEC), in which the CS overlaps the US and the two stimuli are terminated at the same time. It is well established that the brainstem-cerebellar circuit is the essential circuitry for the DEC ${ }^{[1,3-6]}$. In addition, components of the auditory CS pathway (eg, the inferior colliculus or auditory thalamus) have 
recently been added to the DEC circuit ${ }^{[7-14]}$. In contrast, several forebrain structures, such as the medial prefrontal cortex $(\mathrm{mPFC})^{[15-21]}$ and the hippocampus ${ }^{[22-26]}$, are required for TEC in addition to a brainstem-cerebellar circuit ${ }^{[27-31]}$.

It has been reported that electrical stimulations of the several key components in the auditory or visual CS pathway, including the cochlear nucleus ${ }^{[32]}$, medial auditory thalamic ${ }^{[11]}$, auditory cortex ${ }^{[33]}$, lateral geniculate, superior colliculus, visual cortex ${ }^{[34,]}$, pontine nuclei ${ }^{[6,35-37]}$, cerebellar mossy fiber ${ }^{[38,39]}$, parallel fibers $^{[40,41]}$, interpositus nucleus ${ }^{[42,43]}$, etc, can serve as effective CSs for establishing eyeblink conditioning. Moreover, eyeblink conditioning has also been successfully achieved by using stimulation of other brain areas outside of CS pathway, like the primary somatosensory cortex, the coronal-precruciate cortex ${ }^{[44-46]}$, etc. However, less work has been done to examine whether stimulation of the highest level in the hierarchical organization of the mammalian cortex (ie, PFC) is a sufficient CS to support associative eyeblink conditioning.

Cumulative evidence has demonstrated that PFC is implicated in many critical cognitive functions ${ }^{[47-49]}$ and that mPFC is closely involved in associative learning ${ }^{[50]}$ such as eyeblink conditioning $^{[15-18,51]}$. Electrical stimulation of the right rostal PFC of a cat was an effective CS for eyeblink conditioning ${ }^{[52]}$. Given that the caudal mPFC input to the pontine nuclei is necessary for eyeblink conditioning ${ }^{[6]}$, it can be hypothesized that electrical stimulation of the caudal $\mathrm{mPFC}$ as a CS paired with an airpuff US is sufficient for establishing eyeblink conditioning, and that it is dependent on the cerebellar interpositus nucleus. The present study was designed to determine whether electrical stimulation of caudal mPFC is a sufficient CS for establishing eyeblink conditioning, and whether it is dependent on the cerebellar interpositus nucleus. Furthermore, the caudal mPFC play an important role in long TEC (eg, TEC with a 500-ms trace interval, the interval between CS offset and US onset) ${ }^{[6,17,18,53,54]}$, but not in short TEC (eg TEC with a 150-ms trace interval) or DEC ${ }^{[6,17-19,21,55-59]}$. Thus, the current study was also designed to examine the differences in CRs among the three eyeblink conditioning paradigms when the caudal mPFC was selected as the site at which CS stimulation was applied.

\section{Materials and methods Subjects}

A total of 40 adult female albino Dunkin-Hartley guinea pigs were included in the study. The guinea pigs weighed 500-550 g and were approximately 4-5 months old at the time of surgery. Before the experiments and between the conditioning sessions, these animals were individually housed in standard plastic cages that operated on a 12:12 light/dark cycle. The animals were granted free access to food and water ad libitum. The room temperature was maintained at $25 \pm 1^{\circ} \mathrm{C}$. All experiments were performed between 8:00 AM and 6:00 PM during the light portion of the cycle. The experimental procedures were approved by the Animal Care Committee of the Third Military Medical University and were performed in accordance with the principles outlined in the NIH Guide for the Care and Use of Laboratory Animals. All efforts were made to optimize comfort and to minimize the use of the animals.

\section{Surgery}

The animals were allowed to remain undisturbed in their cages for 1 week prior to surgery. The guinea pigs were anesthetized with a mixture of ketamine $(80 \mathrm{mg} / \mathrm{kg}$, ip; Hengrui, Lianyungang, China) and xylazine (5 mg/kg, ip; SigmaAldrich, St Louis, MO, USA). The anesthetized animal's head was secured to a stereotaxic apparatus (SR-6N, Narishige, Tokyo, Japan) with lambda positioned $1.0 \mathrm{~mm}$ ventral to bregma. A longitudinal incision was subsequently made to reveal the skull onto which a Plexiglas headstage $(1.0 \mathrm{~cm} \times$ $1.0 \mathrm{~cm} \times 0.5 \mathrm{~cm})$, designed to secure the animal's head, was cemented with dental cement using four stainless steel anchoring screws. One small hole (diameter: $1.0 \mathrm{~mm}$ ) was drilled on the right side of the skull centered on the right caudal $\mathrm{mPFC}$ at the following stereotaxic coordinates: anteroposterior (AP) $+13.0 \mathrm{~mm}$, mediolateral (ML) $1.0 \mathrm{~mm}$ relative to the frontal zero plane, and the midline sinus, respectively. Then, a stainless steel stimulating electrode (№ 792500, A-M Systems, Sequim, WA, USA; coated diameter: $33.20 \mu \mathrm{m}$, bare diameter: $254.00 \mu \mathrm{m}$ ) was implanted into the right caudal mPFC through the hole according to an atlas of the guinea pig brain ${ }^{[60]}$, and the electrode's tip was directed to the following stereotaxic coordinates: AP+13.0 mm, ML $1.0 \mathrm{~mm}$, dorsoventral (DV)-2.5 $\mathrm{mm}$ to the skull surface (Figure 1A, 1B). Moreover, another small hole (diameter: $1.0 \mathrm{~mm}$ ) was drilled on the left side of the skull centered on the left cerebellar interpositus nucleus at the following stereotaxic coordinates: AP-3.0 mm, ML 2.5 $\mathrm{mm}$ relative to the frontal zero plane, and the midline sinus, respectively. Then, a stainless steel guiding cannula (№ 62001, RWD, Shenzhen, China; external diameter: $0.67 \mathrm{~mm}$, internal diameter: $0.30 \mathrm{~mm}$ ) was implanted into the left cerebellar interpositus nucleus through the hole according to an atlas of the guinea pig brain ${ }^{[60]}$, and its tip was directed to the following stereotaxic coordinates: (AP-3.0 mm, ML $2.5 \mathrm{~mm}$, DV-5.5 mm) (Figure 1A, 1B). The infusion cannula (№ 62201, RWD, Shenzhen, China; external diameter: $0.20 \mathrm{~mm}$, internal diameter: $0.10 \mathrm{~mm}$ ) extended $0.5 \mathrm{~mm}$ beyond the tip of the guiding cannula to the final infusion position at the following stereotaxic coordinates: (AP-3.0 mm, ML $2.5 \mathrm{~mm}, \mathrm{DV}-6.0$ $\mathrm{mm})$. The reference electrode was a copper wire $(0.5 \mathrm{~mm}$ in diameter) attached to the four stainless steel anchoring screws implanted into the skull. This wire was not in direct contact with the skull or brain tissue. To prevent occlusion, a removable stainless steel stylet (No 62101, RWD, Shenzhen, China) was inserted into the guiding cannula. The stylet provided up to $0.5 \mathrm{~mm}$ of extension beyond the tip of the guiding cannula. The stimulating electrode, reference electrode, and guiding cannula were fixed to the skull with dental cement. Finally, a small nylon loop was sutured into but not through the edge of the upper left eyelid. In the present study, this loop was utilized to attach the upper left eyelid to a movement-measuring device. After the surgery, the animals were allowed 1 week of 
A

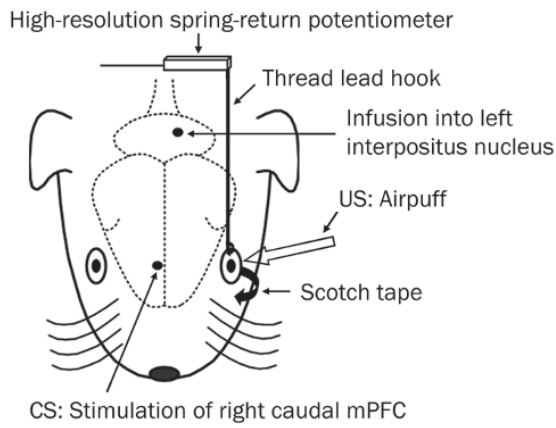

B

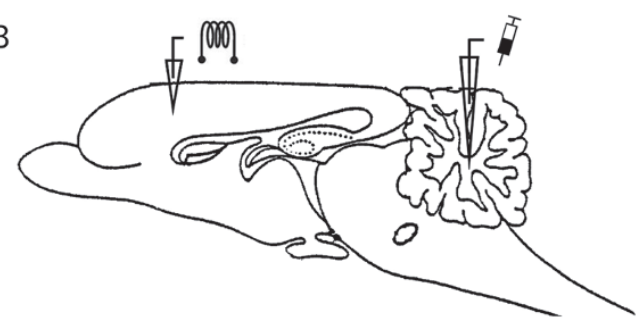

C
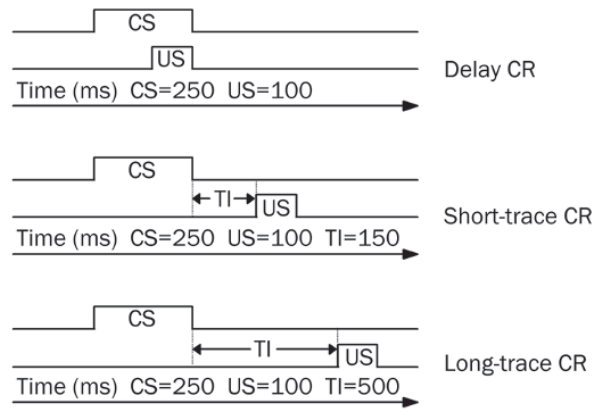

Figure 1. Experimental design. (A) The upper left eyelid movements were measured by a high-resolution spring-return potentiometer that was attached via a thread lead that was hooked through a nylon loop, which was sutured into the left upper eyelid, and the left lower eyelid was taped open. One electrode was implanted in the right caudal medial prefrontal cortex (mPFC) and one infusion guiding cannula was implanted in left cerebellar interpositus nucleus. Electrical stimulation of right caudal mPFC was used as a conditioned stimulus (CS), and airpuff was presented to the left cornea as an unconditioned stimulus (US). (B) Diagram of the sagittal section of guinea pig brain, showing the stimulating and infusion sites. (C) Schematic diagram showing the delay, short-trace, and longtrace eyeblink conditioned response (CR) paradigms used in the present study. Note that the CS, US, and total trial lengths were equal in each conditioning. Short and long-trace CR were different in trace interval (TI) length.

recovery.

\section{Apparatus}

Eyelid movements were measured by a high-resolution spring-return potentiometer (JZ101, XH, Beijing, China) that was attached via a thread lead hooked through the nylon loop sutured into the upper left eyelid. A stimulator (YC-2, Cheng Yi, Chengdu, China) was used to deliver a stimulation CS, while a plastic pipe placed $1.0 \mathrm{~cm}$ from the animal's left eye- ball was used to deliver a corneal airpuff US (Figure 1A). A custom computerized monitoring system controlled presentations of the CS and US. Eyelid-movement mechanograms and applied-stimuli markers were digitized by a data-acquisition system (RM6280C, Cheng Yi, Chengdu, China) at a sample rate of $10 \mathrm{kHz}$ and were acquired using the system's built-in software (v 4.7). A Windows PC was used to store and analyze the behavioral data.

\section{Behavioral procedures}

The 40 guinea pigs were divided into four groups: delay conditioned $(n=10)$, short-trace conditioned $(n=10)$, long-trace conditioned $(n=10)$, and pseudo-conditioned $(n=10)$. Each group represented one training paradigm. Following postoperative recovery, the animals were adapted to the experimental environment for two sessions at $60 \mathrm{~min}$ per session. These two sessions were followed by 10 consecutive daily sessions of acquisition training. Immediately following acquisition training, the three conditioned groups underwent 2 consecutive daily sessions of drug infusion. During the acquisition sessions and the drug infusion sessions, the animals were restrained in a Plexiglas container $(25 \mathrm{~cm} \times 15 \mathrm{~cm} \times 15 \mathrm{~cm})$ located in a soundand light-attenuated chamber, and their heads were secured with blunt ear bars that pressed on the headstages. The left eye of the animal was held open in a confirmable position, with the nylon loop sutured into the left upper eyelid, which was linked to the high-resolution spring-return potentiometer. The voltage level represented the eyeblink baseline position, which was manually calibrated to a constant value. Moreover, the animal's left lower eyelid was taped open. These two measures ensured continual exposure of the left cornea.

During behavioral training, electrical stimulation of the caudal mPFC functioned as the CS, which was a $200-\mathrm{Hz}, 250$ $\mathrm{ms}$ train of monophasic pulses (cathodal, square, current level of 50-200 $\mu \mathrm{A}$, a pulse duration of $0.1 \mathrm{~ms}$ ). The electrical stimulation parameters were chosen based on the recent studies $^{[8,11,14,58]}$. The stimulation intensity for each guinea pig was set carefully before training by increasing the test current until a behavioral response was observed to avoid biasing the experimental results through an electrical startle response and a spreading of the electrical stimulation current to remote brain areas, such as hippocampus, premotor cortex, and somatosensory cortex. The current was then turned down in $5-\mu \mathrm{A}$ increments until there was no observable behavioral response. Typical behavioral responses observed from the test stimulation included movements of the eyelid, eye, ear and/ or head ${ }^{[8,11,14,58]}$. In most of the cases $(28 / 37)$ in the present experiment, the threshold stimulation was between 50-120 $\mu \mathrm{A}$. An additional evoked field potentials recording test showed that the caudal mPFC stimulation with $200 \mu \mathrm{A}$ or below did not evoke any field potential in the motor cortex, somatosensory cortex, or the cerebellar cortex (date not shown). The US was a 100-ms, 3.0-psi airpuff. A daily acquisition training session consisted of five 10-trial blocks. However, a daily infusion session (d 11 and d 12) consisted of three 10-trial blocks before the infusion and five 10-trial blocks after the infusion. 
Moreover, each block comprised nine CS-US paired trials and one CS alone trial. The trials were separated by a variable intertrial interval of 20-40 s with a mean intertribal interval of $30 \mathrm{~s}$. For the delay-conditioning paradigm, the US terminated simultaneously with the offset of the CS (the interstimulus interval was $150 \mathrm{~ms}$ ). For the short-trace and long-trace conditioning paradigms, a stimulus-free trace interval of $150 \mathrm{~ms}$ or $500 \mathrm{~ms}$ was interposed between the CS termination and the US onset, respectively (Figure 1C). For the pseudo-conditioning paradigm, the US was presented at a random interval between 1 and $10 \mathrm{~s}$ after the CS onset. All experiments were performed during the light phase of the light/dark cycle.

\section{Drug infusions}

Two drug infusion sessions were conducted during d 11 and d 12. Each infusion session began with three blocks of training to establish a baseline of response prior to each drug infusion. Drug infusions were performed $20 \mathrm{~min}$ before the subsequent beginning of conditioning training. Muscimol (Sigma-Aldrich, St Louis, MO, USA), which produces inactivation only to the soma of neurons but not to the fibers of passage, was dissolved in saline (phosphate buffer, $\mathrm{pH}$ 7.4), which served as the vehicle prior to use. During the first infusion session (d 11), $1.0 \mu \mathrm{g}$ muscimol in $1.0 \mu \mathrm{L}$ saline was infused into the guinea pigs' left cerebellar interpositus nucleus. Infusion procedures for each animal included removal of the internal stylet from the guiding cannula, insertion of a stainless steel infusion cannula that extended $0.5 \mathrm{~mm}$ below the tip of the guiding cannula, infusion of the drug at $0.5 \mu \mathrm{L} / \mathrm{min}$ via polyethylene tubing connected to a microsyringe, removal of the needle 5 min after the cessation of infusion, and finally, reinsertion of the internal stylet. During the second infusion session (d 12), $1.0 \mu \mathrm{L}$ saline was infused into the guinea pigs' left cerebellar interpositus nucleus. The drug infusion procedures used in the second infusion session were the same as those used in the first infusion session. All of the animals were allowed $24 \mathrm{~h}$ to recover between the infusion sessions. Several prior studies that used similar infusion procedures have reported that muscimol spread maximally within the 10-20 min following infusion, that the effective inactivation radius was $1.5-2.0 \mathrm{~mm}$, and that the blocking effect persisted for a period of up to $2.0 \mathrm{~h}$ in both cortical and subcortical tissues ${ }^{[61-63]}$. However, in present study, the exact drug diffusion into each animal is unknown because the spread of the three drugs was not measured directly.

\section{Histology}

After the completion of behavioral experiments, all of the animals were given a lethal dose of pentobarbital sodium (150 mg/kg, ip; SCRC, Shanghai, China) and were perfused transcardially with physiological saline followed by $4 \%$ paraformaldehyde, which was prepared in phosphate-bufferer $(0.1 \mathrm{~mol} / \mathrm{L}, \mathrm{pH}$ 7.4). The brains were removed from the skulls and stored in $4 \%$ paraformaldehyde for several days. Four days prior to sectioning, the brains were transferred to a $30 \%$ sucrose $4 \%$ paraformaldehyde solution. Frozen coronal sections measuring $30 \mu \mathrm{m}$ in thickness were taken from the sites of the electrode and infusion cannula implantation. The slices were stained with cresyl violet. The locations of the electrode and infusion cannula tips within the brains were carefully determined using a light microscope (SMZ1500, Nikon, Tokyo, Japan) with a digital camera (DXM1200F, Nikon, Tokyo, Japan) and were drawn onto plates using a stereotaxic atlas of the guinea pig brain ${ }^{[60]}$.

\section{Behavioral data analysis}

For each training trial, 2000-ms time periods were recorded during the conditioning trials and 12-s time periods were recorded during the pseudo-conditioning trials beginning $800 \mathrm{~ms}$ before the onset of the CS. Drug infusions were not recorded. All data presented in this paper are measurements of the left upper eyelid movements. The parameters of eyeblink responses were analyzed using custom software.

Each CS-US paired trial presented during the conditioning training was subdivided into three discontinuous analysis periods: (1) a "baseline" period, which occurred at 0-800 ms before the CS onset; (2) a "CR" period, which occurred at 140 ms before the US onset; and (3) a "UR" period, which occurred at 0-250 ms after the US onset. The "baseline" period and "CR" period of each CS-alone trial were divided in the same manner as the baseline and CR periods from the CS-US paired trials for the same training paradigm. A significant eyelid movement was defined as an increase in mechanogram amplitude that was greater than the mean baseline amplitude and had four times the standard deviation of the baseline activity. In addition, the significant eyelid movement required a minimal duration of $15 \mathrm{~ms}$. Any significant eyelid movement during the latter two periods defined above was counted as a CR or a $\mathrm{UR}$, respectively. The percentage of $\mathrm{CR}(\mathrm{CR} \%)$ was defined as the ratio of the number of trials containing the CR to the total number of valid trials. The CR peak amplitude was defined as the maximum amplitude change from baseline during the $\mathrm{CR}$ period. The trials containing $\mathrm{CR}$ were selected for analysis of $\mathrm{CR}$ peak amplitude. The $\mathrm{CR}$ relative peak latency was defined as the time interval from the CR peak to the US onset.

Only the CR\% was analyzed for the animals that received pseudo-conditioning training. For each trial, a significant eyelid movement that occurred within the time period 140 $\mathrm{ms}$ before the US onset was defined as a CR-like eyeblink response.

\section{Statistical analysis}

All data were expressed as means \pm SEM. Statistical significance was determined by a least significant difference (LSD) post-hoc test following a two-way repeated measures analyses of variance (ANOVA), a separate one-way repeated measures ANOVA, or a separate one-way ANOVA using the SPSS software for Windows package (v 18.0). A value of $P<0.05$ was considered statistically significant. 


\section{Results}

Electrode and infusion cannula tips placements

Placement of the electrode cannula and infusion tips was carefully checked before the behavioral analysis commenced. An animal's data were excluded from the analysis if the electrode tip was not in the right caudal $\mathrm{mPFC}$ or the infusion cannula tip was not in the left cerebellar interpositus nucleus. Electrode tip placements in the right caudal $\mathrm{mPFC}$ were verified by examining a series of coronal sections. All electrode tips were placed in the right caudal mPFC $(n=37)$. The infusion cannula tip placements in the left cerebellar interpositus nucleus were also verified by examining a series of coronal sections (Figure $2 \mathrm{~A})$. Most of the infusion cannula tip placements were in or near the left cerebellar interpositus nucleus $(n=35)$ with one exception each in the delay and short-trace conditioned group (Figure 2B).
A

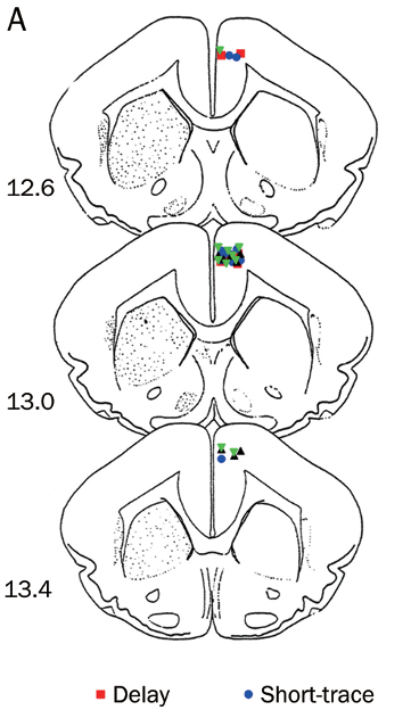

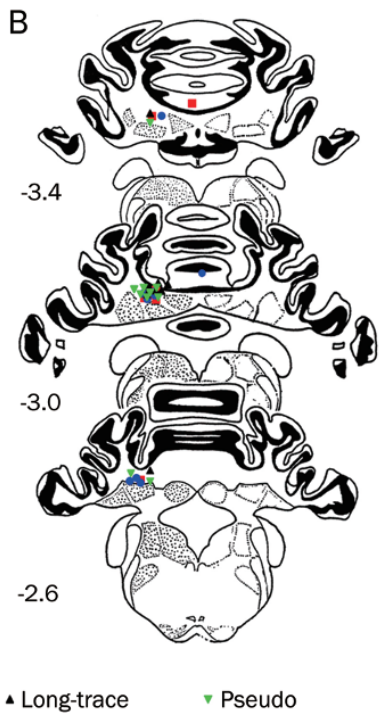

B

$\triangle$ Long-trace $\quad$ Pseudo
Figure 2. Histological reconstructions of the electrode and infusion cannula tips. Schematic illustration of the locations of all electrode tips (A) and all infusion cannula tips (B) for the delay $(\boldsymbol{\square} ; n=9)$, short-trace $(\bullet$; $n=10)$, long-trace $(\mathbf{\Lambda} ; n=8)$, and pseudo conditioned $(\boldsymbol{\nabla} ; n=10)$ groups, respectively. Note that one infusion cannula tip of the delay conditioned group and one infusion cannula tip of the short-trace conditioned group were not in or near the left cerebellar interpositus nucleus. Numbers to the left represent distance $(\mathrm{mm})$ from the frontal zero plane. The coronal brain plates are adapted from the atlas of Rapisarda and Bacchelli (1977).

\section{Acquisition of eyeblink conditioning by the guinea pigs}

The data from two animals in the delay-conditioned group, from one animal in the short-trace conditioned group and from two animals in long-trace conditioned group were removed from the analysis, because either the infusion cannula tips placements were not in or near the left cerebellar interpositus nucleus $(n=2)$ or the animal had died before the end of the experiment $(n=3)$. The $\mathrm{CR} \%$ increased as a function of sessions for the delay, short-trace and long-trace conditioned groups (Figure 3A). This increase was confirmed by a twoway repeated measures ANOVA, there was a significant interaction between groups and sessions $\left[F_{(27,279)}=7.289, P<0.001\right]$, and significant main effects of group $\left[F_{(3,31)}=39.295, P<0.001\right]$ and session $\left[F_{(9,279)}=38.940, P<0.001\right]$. Furthermore, LSD posthoc tests revealed that the $\mathrm{CR} \%$ of the delay, short-trace, and long-trace conditioned groups was significantly higher than the $\mathrm{CR} \%$ of the pseudo-conditioned group $(P<0.001, P<0.001$, and $P=0.026$, respectively; Figure $3 \mathrm{~A})$. The simple main effects of session for $\mathrm{CR} \%$ during acquisition training were further analyzed using separate one-way repeated measures ANOVA.
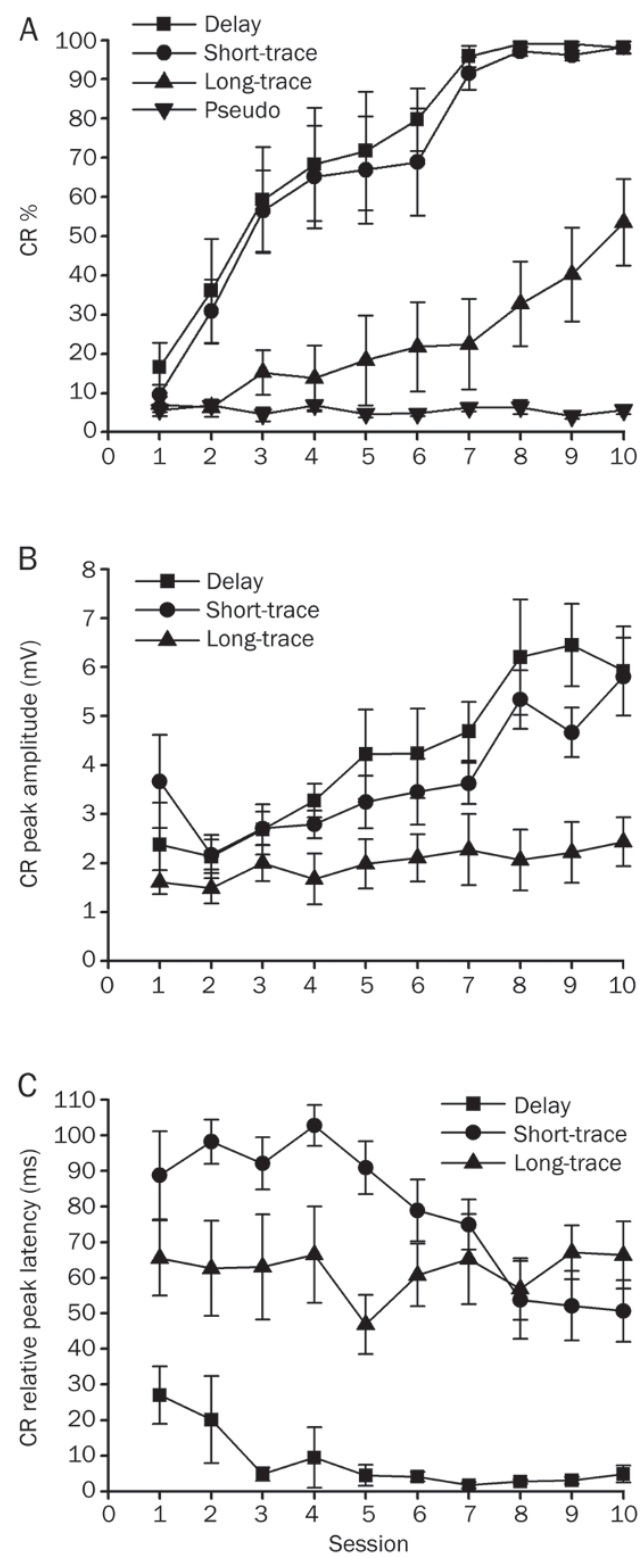

Figure 3. Acquisition of the eyeblink conditioned responses (CR) for delay $(n=8)$, short-trace $(n=9)$, long-trace $(n=8)$, and pseudo $(n=10)$ conditioned groups given training with a right caudal mPFC conditioned stimulus (CS) across 10 acquisition training sessions. (A) CR percentage, (B) CR peak amplitude, and (C) $C R$ relative peak latency are given as mean \pm standard error (SEM). Error bars represent the SEM. 
This analysis revealed that the simple main effects of session on $\mathrm{CR} \%$ were significant for the delay $\left[F_{(9,63)}=14.517, P<0.001\right]$, short-trace $\left[F_{(9,72)}=2.332, P<0.001\right]$, and long-trace $\left[F_{(9,63)}=5.543\right.$, $P<0.001]$ conditioned groups, but not for the pseudo-conditioned group $\left[F_{(9,81)}=0.626, P=0.772\right.$; Figure $\left.3 \mathrm{~A}\right]$.

To investigate the effects of the different conditioning training paradigms on the CR pattern, CR peak amplitude and relative peak latency was analyzed for all of the animals. LSD post-hoc tests confirmed that the CR peak amplitude in the long-trace conditioned group was significantly lower than that in the delay $(P<0.001)$ and short-trace $(P=0.002)$ conditioned groups. The latter two groups did not differ significantly from each other $(P=0.356$; Figure $3 \mathrm{~B})$. In addition, a separate oneway repeated measures ANOVA revealed that the simple main effects of session on the CR peak amplitude were significant for both the delay $\left[F_{(9,63)}=5.143, P<0.001\right]$ and shorttrace $\left[F_{(9,72)}=4.529, P<0.001\right]$ conditioned groups, but not for the long-trace conditioned group $\left[F_{(9,81)}=0.873, P=0.554\right.$; Figure 3B].

Furthermore, LSD post-hoc tests revealed that the CR relative peak latency in the delay conditioned group was significantly lower than that in either the short-trace $(P<0.001)$ or the longtrace $(P<0.001)$ conditioned groups. The latter two groups differed significantly from each other $(P=0.019$; Figure $3 C)$. Moreover, the LSD post-hoc tests confirmed that the CR relative peak latency during session 1 was significantly higher than during session 10 for the delay $(P=0.041)$ and short-trace $(P=0.043)$ conditioned groups, but not for the long-trace conditioned group $(P=0.994$; Figure $3 C)$.

\section{Effects of muscimol infusion into the left cerebellar interpositus nucleus on DEC expression}

Infusion of muscimol into the left cerebellar interpositus nucleus significantly decreased the CR\% (Figure 4A, right panel) and the $C R$ peak amplitude for the group that received delay conditioning (Figure $4 \mathrm{~B}$, right panel). A twoway repeated measures ANOVA performed on CR\% (Figure $4 \mathrm{~A}$, left panel) and CR peak amplitude (Figure $4 \mathrm{~B}$, left panel) revealed there was no significant effect of trial block $\left[F_{(1,14)}=0.299, P=0.593\right.$ and $F_{(1,14)}=0.028, P=0.870$, respectively] during the pre-infusion block (trial blocks 1-3). However, the two-way repeated measures ANOVA confirmed that the CR\% (Figure 4A, right panel) and the CR peak amplitude (Figure $4 \mathrm{~B}$, right panel) for the animals that received the muscimol infusion were significantly lower than those observed for the animals that received the saline infusion $\left[F_{(1,14)}=25.599, P<0.001\right.$ and $F_{(1,14)}=36.147, P<0.001$, respectively] during the post-infusion block (trial blocks 4-8).

\section{Effects of muscimol infusion into the left cerebellar interpositus} nucleus on short TEC expression

Infusion of muscimol into the left cerebellar interpositus nucleus significantly decreased the CR\% (Figure 5A, right panel) and the CR peak amplitude for the group that received short-trace conditioning (Figure 5B, right panel). A twoway repeated measures ANOVA performed on $\mathrm{CR} \%$ (Figure $5 \mathrm{~A}$, left panel) and CR peak amplitude (Figure 5B, left panel)
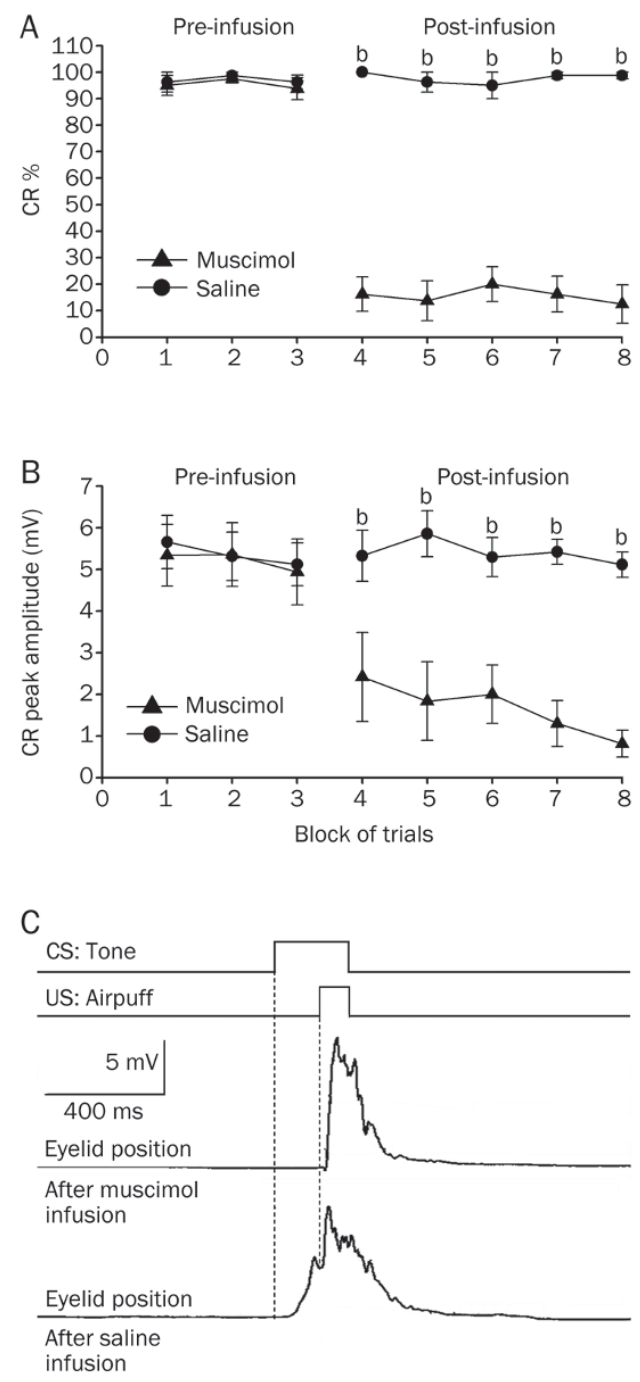

Figure 4. Delay conditioned group data for the effects of muscimol ( $\mathbf{\Delta})$ and saline $(\bullet)$ infused into the left cerebellar interpositus nucleus. (A) Infusion of muscimol abolished the responses almost completely as illustrated by its effects on the percentage of trials in which the delay CRs were seen, whereas infusion of saline had no significant effect on the delay CRs. (B) Muscimol infusion significantly decreased the peak amplitude of the delay CRs. (C) Eyelid position of an animal after muscimol and saline infusion in the sixth trial. Upper panel: the conditioning paradigm illustrating the timing of the CS and the US. Middle panel: eyelid position after muscimol infusion. Lower panel: eyelid postion after saline infusion. All data are from the same animals. Mean $\pm S E M$. $n=8$. ${ }^{\mathrm{b}} \mathrm{P}<0.05$ vs control.

revealed that there was no significant effect of trial block $\left[F_{(1,16)}=0.250, P=0.624\right.$ and $F_{(1,16)}=0.516, P=0.483$, respectively $]$ during the pre-infusion block (trial blocks 1-3). However, the two-way repeated measures ANOVA confirmed that the CR\% (Figure 5A, right panel) and the CR peak amplitude (Figure 5B, right panel) for the animals that received the muscimol infusion were significantly lower than for the animals that received the saline infusion $\left[F_{(1,16)}=72.147, P<0.001\right.$ and $F_{(1,16)}=33.558, P<0.001$, respectively] during the post-infusion 

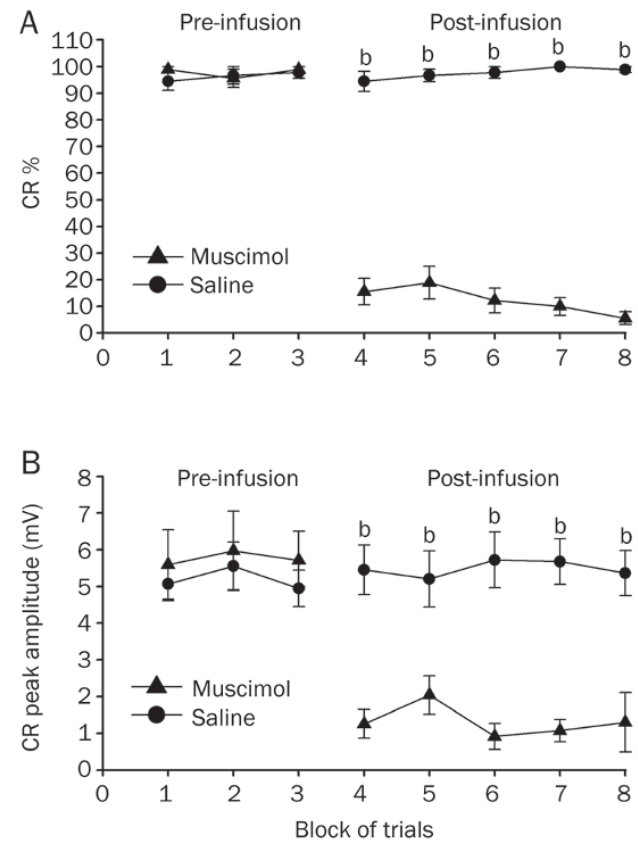

C

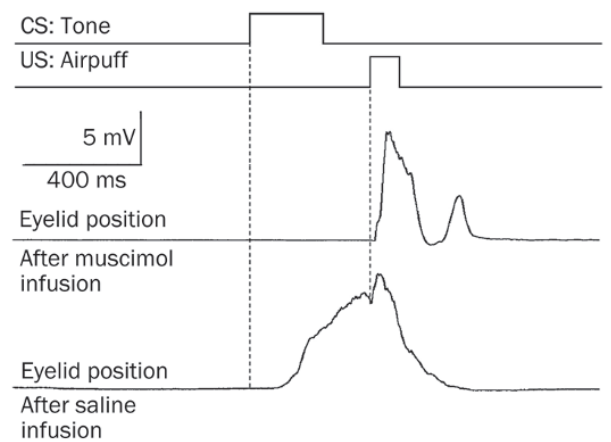

Figure 5. Short-trace conditioned group data for the effects of muscimol $(\boldsymbol{\Delta})$, and saline $(\bullet)$ infused into the left cerebellar interpositus nucleus. (A) Infusion of muscimol abolished the responses almost completely as illustrated by its effects on the percentage of trials in which the shorttrace $C R s$ are seen, whereas infusion of saline had no significant effect on the short-trace CRs. (B) Muscimol infusion significantly decreased the peak amplitude of the short-trace CRs. (C) Eyelid position of an animal after muscimol and saline infusion in the sixth trial. Upper panel: the conditioning paradigm illustrating the timing of the CS and the US. Middle panel: eyelid position after muscimol infusion. Lower panel: eyelid postion after saline infusion. All data are from the same animals. Mean \pm SEM. $n=9 .{ }^{\mathrm{b}} P<0.05$ vs control.

block (trial blocks 4-8).

\section{Effects of muscimol infusion into the left cerebellar interpositus} nucleus on long TEC expression

Infusion of muscimol into the left cerebellar interpositus nucleus significantly decreased the $\mathrm{CR} \%$ (Figure $6 \mathrm{~A}$, right panel) and the CR peak amplitude (Figure 6B, right panel) for the group that received long-trace conditioning. A twoway repeated measures ANOVA performed on CR\% (Figure
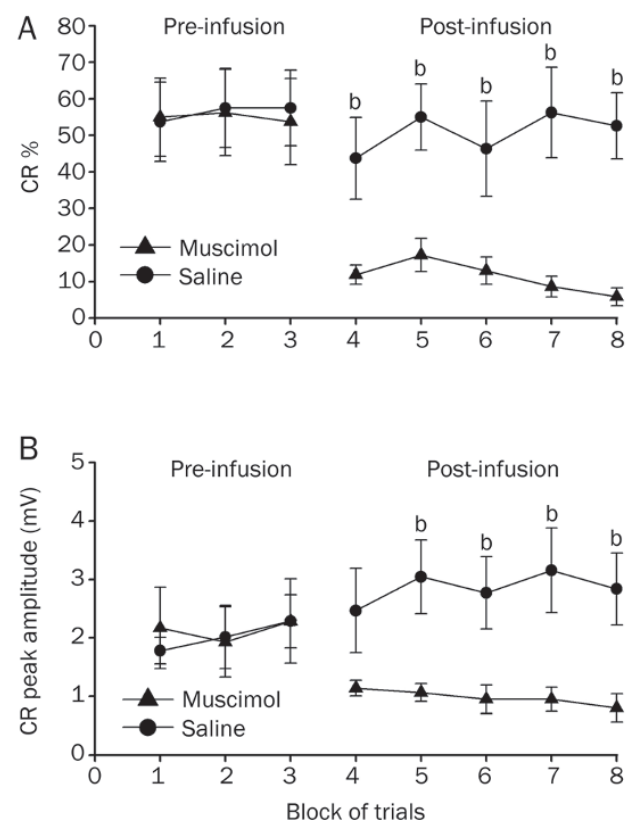

C

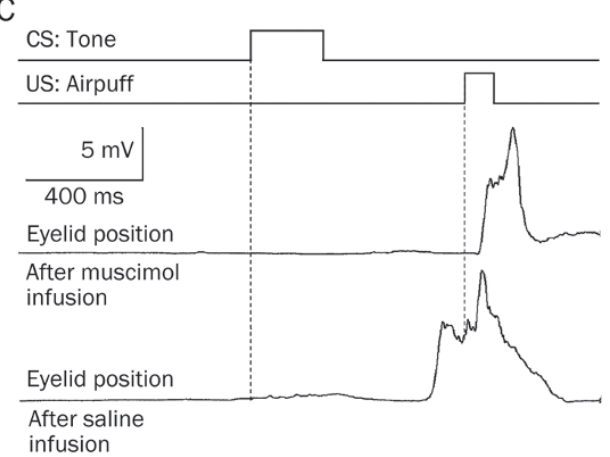

Figure 6. Long-trace conditioned group data for the effects of muscimol $(\boldsymbol{\Delta})$ and saline $(\bullet)$ infused into the left cerebellar interpositus nucleus. (A) Infusion of muscimol abolished the responses almost completely as illustrated by its effects on the percentage of trials in which the longtrace CRs are seen, whereas infusion of saline had no significant effect on the long-trace CRs. (B) Muscimol infusion had significant effect on peak amplitude of the long-trace CRs. (C) Eyelid position of an animal after muscimol and saline infusion in the sixth trial. Upper panel: the conditioning paradigm illustrating the timing of the CS and the US. Middle panel: eyelid position after muscimol infusion. Lower panel: eyelid postion after saline infusion. All data are from the same animals. Mean \pm SEM. $n=8$. ${ }^{\mathrm{b}} P<0.05$ vs control.

6A, left panel) and CR peak amplitude (Figure 6B, left panel) revealed that there was no significant effect of trial block $\left[F_{(1,14)}=0.007, P=0.933\right.$ and $F_{(1,14)}=0.089, P=0.770$, respectively] during the pre-infusion block (trial blocks 1-3). In contrast, the two-way repeated measures ANOVA confirmed that the $\mathrm{CR} \%$ (Figure 6A, right panel) and the $\mathrm{CR}$ peak amplitude (Figure 6B, right panel) for the animals that received the muscimol infusion were significantly lower than for the animals that received the saline infusion $\left[F_{(1,14)}=25.801, P<0.001\right.$ and 
$F_{(1,14)}=8.313, P<0.001$, respectively] during the post-infusion block (trial blocks 4-8).

\section{Discussion}

In the initial portion of this study, it was demonstrated that electrical stimulation of the caudal MPFC as a CS paired with an airpuff US was sufficient for establishing eyeblink conditioning in guinea pigs. In contrast, unpaired presentations of the stimulation CS and airpuff US did not obtain CR across $10 \mathrm{~d}$ of training, suggesting that CRs observed in the conditioned groups were due to associative learning. Against our expectations, the guinea pigs acquired long TEC slower than both DEC and short TEC, in agreement with the previous studies using peripheral CS (eg, a tone or a light CS) in eyeblink conditioning ${ }^{[64,65]}$. There is a possibility that the longer trace interval between the CS and the US makes it difficult for them to timely converge inside brain, and that additional brain regions such as the hippocampus, $\mathrm{MPFC}$ and amygdala are required for establishment of long TEC ${ }^{[6,13,17,18,22-24,53,54,66-69]}$. There were significant increases in CR amplitudes and decreases in CR relative peak latency in both delay and shorttrace, but not in long-trace conditioned groups, attributing to that long TEC had not been well learned during successive 10 sessions. Moreover, an additional evoked field potentials recording test showed that the caudal $\mathrm{MPFC}$ stimulation with $200 \mu \mathrm{A}$ or below did not evoke any field potential in the motor cortex, somatosensory cortex, or the cerebellar cortex. Thus, the current results provide direct support for our hypothesis that electrical stimulation of MPFC as a CS paired with an airpuff US is sufficient for establishing eyeblink conditioning.

In the second portion of this study, infusion of muscimol into the left interpositus nucleus impaired CRs expression in the three conditioned groups, suggesting that the interpositus nucleus is critical for expression of this special CR. It is consistent with the findings of the previous studies using peripheral CS in eyeblink conditioning that cerebellar interpositus nucleus plays an essential role in CR expression ${ }^{[4,27,29,31,66,70,71]}$. Steinmetz ${ }^{[72]}$ speculated that the cerebellum may only be involved in simple, discrete, aversive, and somatic associative learning that occurs with a relatively short interstimulus interval. The current results support his speculation, and show that the expression of this special CR, which is also a simple, discrete, somatic, aversive, and defensive behavior induced by stimulation of caudal mPFC as a CS, is still dependent on the cerebellum. The results from the present study further imply that it is the features of the CR rather than the CS that decide whether the cerebellum is necessary for $\mathrm{CR}$ operation.

Given that $\mathrm{mPFC}$ is necessary for long TEC ${ }^{[6,17,18,53,54]}$, but not for DEC and short TEC ${ }^{[6,17-19,21,55-59]}$, it is expected that long TEC would be acquired more rapidly than DEC and short TEC at least in some manner when stimulation of the caudal $\mathrm{mPFC}$, part of the long TEC circuit, was used as a CS. However, the present results show that the guinea pigs acquired DEC and short TEC more rapidly than long TEC. The current findings combined with the results of previous studies may indicate that one brain area at which an effective and sufficient stimula- tion CS for establishing CR was applied can not be interpreted as an essential area for classical eyeblink conditioning. For instance, lesions of the visual cortex did not prevent acquisition of CRs with a light $\mathrm{CS}^{[73]}$, suggesting that the visual cortex is not involved in the process of $\mathrm{CR}$ acquisition, whereas stimulation of the visual cortex can be successfully used as a $\mathrm{CS}$ to establish $\mathrm{CR}^{[34]}$. Moreover, although lesions of the pretectal nuclei ${ }^{[73]}$ and hippocampus ${ }^{[22-24,68]}$ retarded acquisition of eyeblink conditioning, stimulation of the anterior pretectal nucleus ${ }^{[1]]}$ and of the CA1 layer of hippocampus ${ }^{[7]}$ can not be served as effective CSs for establishing eyeblink conditioning. Therefore, the present results only suggest that electrical stimulation of $\mathrm{mPFC}$ is a very effective and sufficient CS for establishing eyeblink conditioning, and that it is dependent on the cerebellar interpositus nucleus, but can not be interpreted as providing evidence that $\mathrm{MPFC}$ is critically involved in DEC, short TEC, or long TEC.

It is worth noting that long TEC with a tone CS acquisition requires $\mathrm{mPFC}$ that persists mossy fiber activity through the stimulus-free trace interval to overlap in time with the

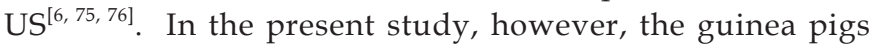
could develop long TEC to MPFC stimulation CS even when it did not co-terminate with the US. There is a possibility that the MPFC activity which begins during the stimulation CS and persists beyond the stimulation CS offset to overlap with the US may induce long TEC successful establishment. Indeed, it is reported that the activity in the neurons of mPFC began during the tone CS and persisted to overlap with the US during TEC ${ }^{[77]}$.

Despite careful and detailed analysis of the CRs acquisition to electrical CSs by previous studies, it is difficult to establish a minimum range of current for the electrical CS which is required for obtaining CR. The threshold stimulation of CS required to establish the $\mathrm{CR}$ in the present study was lower than that in most previously studies $(50-200 \mu \mathrm{A}$ in current study vs $180-250 \mu \mathrm{A}$ for visual cortex stimulation ${ }^{[34]}$ ). This difference may in part due to differences in current spread at different sites of stimulation, but may also relate to differences in the numbers of cells which must be excited for the CS to be effective ${ }^{[45]}$

One of our primary purposes here was to test the effects of stimulation of the caudal mPFC on CR establishment. Whether or not stimulation of other areas of PFC can obtain the $\mathrm{CR}$ is an important issue, and should be investigated in future studies. While the distributed pattern of activation induced by electrical stimulation in awake animals is currently unknown ${ }^{[78]}$, an additional evoked field potentials recording test showed that the caudal mPFC stimulation with $200 \mu \mathrm{A}$ or below did not evoke any field potential in the motor cortex, somatosensory cortex, or the cerebellar cortex. Thus, the present experiment could rule out the possibility that stimulation of the caudal mPFC significantly activates other areas of cortex. In addition, the lesion and field potential data suggest that the lateral pontine nuclei conveys necessary CS signals to the cerebellum in eyeblink conditioning ${ }^{[79,80]}$. Recent studies suggest that mossy fiber activity driven by input from mPFC 
to lateral pontine nuclei which persists through the stimulusfree trace interval to overlap in time with the US supports the auditory TEC ${ }^{[6,75,76]}$. Therefore, the lateral pontine nuclei may also convey the electrical CS signals to the cerebellum in the three types of eyeblink conditioning induced by the electrical stimulation of caudal mPFC. However, this hypothesis needs further testing.

In conclusion, the results from this study show that electrical stimulation of caudal mPFC was a very effective CS for establishing eyeblink conditioning, and that the CR is dependent on the cerebellar interpositus nucleus. Moreover, the guinea pigs acquired delay and short-trace CRs more rapidly than long-trace CR. However, the current results should not be interpreted as providing evidence that $\mathrm{MPFC}$ is involved in DEC, short TEC, or long TEC.

\section{Acknowledgements}

This work was supported by Grants from the National Natural Science Foundation of China (№ 30771769). We thank the assistance of De-ying CHEN in preparing the photomicrographs.

\section{Author contribution}

Jian-feng SUI and Guang-yan WU designed research; Guangyan WU, Juan YAO, Zheng-li FAN, and Lang-qian ZHANG performed research; Juan YAO, Xuan LI, Chuang-dong ZHAO, and Zhen-hua ZHOU analyzed data; Jian-feng SUI and Guang-yan WU wrote the paper.

\section{References}

1 Christian KM, Thompson RF. Neural substrates of eyeblink conditioning: acquisition and retention. Learn Mem 2003; 10: 427-55.

2 Christian KM, Thompson RF. Long-term storage of an associative memory trace in the cerebellum. Behav Neurosci 2005; 119: 52637.

3 Thompson RF. In search of memory traces. Annu Rev Psychol 2005; 56: 1-23.

4 Weeks AC, Connor S, Hinchcliff R, LeBoutillier JC, Thompson RF, Petit TL. Eye-blink conditioning is associated with changes in synaptic ultrastructure in the rabbit interpositus nuclei. Learn Mem 2007; 14: 385-9.

5 Green JT, Arenos JD. Hippocampal and cerebellar single-unit activity during delay and trace eyeblink conditioning in the rat. Neurobiol Learn Mem 2007; 87: 269-84.

6 Kalmbach BE, Ohyama T, Kreider JC, Riusech F, Mauk MD. Interactions between prefrontal cortex and cerebellum revealed by trace eyelid conditioning. Learn Mem 2009; 16: 86-95.

7 Halverson HE, Lee I, Freeman JH. Associative plasticity in the medial auditory thalamus and cerebellar interpositus nucleus during eyeblink conditioning. J Neurosci 2010; 30: 8787-96.

8 Halverson HE, Freeman JH. Medial auditory thalamic input to the lateral pontine nuclei is necessary for auditory eyeblink conditioning. Neurobiol Learn Mem 2010; 93: 92-8.

9 Halverson HE, Poremba A, Freeman JH. Medial auditory thalamus inactivation prevents acquisition and retention of eyeblink conditioning. Learn Mem 2008; 15: 532-8.

10 Freeman $\mathrm{JH}$, Halverson HE, Hubbard EM. Inferior colliculus lesions impair eyeblink conditioning in rats. Learn Mem 2007; 14: 842-6.
11 Campolattaro MM, Halverson HE, Freeman JH. Medial auditory thalamic stimulation as a conditioned stimulus for eyeblink conditioning in rats. Learn Mem 2007; 14: 152-9.

12 Halverson HE, Freeman JH. Medial auditory thalamic nuclei are necessary for eyeblink conditioning. Behav Neurosci 2006; 120: 880-7.

13 Oswald BB, Knuckley B, Maddox SA, Powell DA. Ibotenic acid lesions to ventrolateral thalamic nuclei disrupts trace and delay eyeblink conditioning in rabbits. Behav Brain Res 2007; 179: 111-7.

14 Halverson HE, Freeman $\mathrm{JH}$. Ventral lateral geniculate input to the medial pons is necessary for visual eyeblink conditioning in rats. Learn Mem 2010; 17: 80-5.

15 Simon B, Knuckley B, Churchwell J, Powell DA. Post-training lesions of the medial prefrontal cortex interfere with subsequent performance of trace eyeblink conditioning. J Neurosci 2005; 25: 10740-6.

16 Oswald BB, Maddox SA, Powell DA. Prefrontal control of trace eyeblink conditioning in rabbits: role in retrieval of the CR? Behav Neurosci 2008; 122: 841-8.

17 Oswald BB, Maddox SA, Tisdale N, Powell DA. Encoding and retrieval are differentially processed by the anterior cingulate and prelimbic cortices: a study based on trace eyeblink conditioning in the rabbit. Neurobiol Learn Mem 2010; 93: 37-45.

18 Powell DA, Churchwell J, Burriss L. Medial prefrontal lesions and Pavlovian eyeblink and heart rate conditioning: effects of partial reinforcement on delay and trace conditioning in rabbits (Oryctolagus cuniculus). Behav Neurosci 2005; 119: 180-9.

19 Weible AP, McEchron MD, Disterhoft JF. Cortical involvement in acquisition and extinction of trace eyeblink conditioning. Behav Neurosci 2000; 114: 1058-67.

20 Weible AP, Weiss C, Disterhoft JF. Activity profiles of single neurons in caudal anterior cingulate cortex during trace eyeblink conditioning in the rabbit. J Neurophysiol 2003; 90: 599-612.

21 Takehara-Nishiuchi K, Kawahara S, Kirino Y. NMDA receptor-dependent processes in the medial prefrontal cortex are important for acquisition and the early stage of consolidation during trace, but not delay eyeblink conditioning. Learn Mem 2005; 12: 606-14.

22 Weiss C, Bouwmeester H, Power JM, Disterhoft JF. Hippocampal lesions prevent trace eyeblink conditioning in the freely moving rat. Behav Brain Res 1999; 99: 123-32.

23 Beylin AV, Gandhi CC, Wood GE, Talk AC, Matzel LD, Shors TJ. The role of the hippocampus in trace conditioning: temporal discontinuity or task difficulty? Neurobiol Learn Mem 2001; 76: 447-61.

24 Tseng W, Guan R, Disterhoft JF, Weiss C. Trace eyeblink conditioning is hippocampally dependent in mice. Hippocampus 2004; 14: 5865.

25 Solomon PR, Vander Schaaf ER, Thompson RF, Weisz DJ. Hippocampus and trace conditioning of the rabbit's classically conditioned nictitating membrane response. Behav Neurosci 1986; 100: 72944.

26 Moyer JR Jr, Deyo RA, Disterhoft JF. Hippocampectomy disrupts trace eye-blink conditioning in rabbits. Behav Neurosci 1990; 104: 24352.

$27 \mathrm{Hu} \mathrm{B}$, Yang L, Huang LS, Chen H, Zeng Y, Feng H, et al. Effect of cerebellar reversible inactivations on the acquisition of trace conditioned eyeblink responses in guinea pigs: comparison of short and long trace intervals. Neurosci Lett 2009; 459: 41-5.

$28 \mathrm{Hu} \mathrm{B}$, Lin X, Huang LS, Yang L, Feng H, Sui JF. Involvement of the ipsilateral and contralateral cerebellum in the acquisition of unilateral classical eyeblink conditioning in guinea pigs. Acta Pharmacol Sin 2009; 30: 141-52.

29 Pakaprot N, Kim S, Thompson RF. The role of the cerebellar inter- 
positus nucleus in short and long term memory for trace eyeblink conditioning. Behav Neurosci 2009; 123: 54-61.

30 Takehara K, Kawahara S, Kirino Y. Time-dependent reorganization of the brain components underlying memory retention in trace eyeblink conditioning. J Neurosci 2003; 23: 9897-905.

31 Woodruff-Pak DS, Lavond DG, Thompson RF. Trace conditioning: abolished by cerebellar nuclear lesions but not lateral cerebellar cortex aspirations. Brain Res 1985; 348: 249-60.

32 Freeman JH, Duffel JW. Eyeblink conditioning using cochlear nucleus stimulation as a conditioned stimulus in developing rats. Dev Psychobiol 2008; 50: 640-6.

33 Knowlton BJ, Thompson RF. Conditioning using a cerebral cortical conditioned stimulus is dependent on the cerebellum and brain stem circuitry. Behav Neurosci 1992; 106: 509-17.

34 Halverson HE, Hubbard EM, Freeman JH. Stimulation of the lateral geniculate, superior colliculus, or visual cortex is sufficient for eyeblink conditioning in rats. Learn Mem 2009; 16: 300-7.

35 Freeman JH Jr, Rabinak CA. Eyeblink conditioning in rats using pontine stimulation as a conditioned stimulus. Integr Physiol Behav Sci 2004; 39: 180-91.

36 Rosen DJ, Steinmetz JE, Thompson RF. Classical discrimination conditioning of the rabbit's eyelid response using pontine stimulation as a conditioned stimulus. Behav Neural Biol 1989; 52: 51-62.

37 Steinmetz JE, Lavond DG, Thompson RF. Classical conditioning in rabbits using pontine nucleus stimulation as a conditioned stimulus and inferior olive stimulation as an unconditioned stimulus. Synapse 1989; 3: 225-33.

38 Steinmetz JE, Rosen DJ, Chapman PF, Lavond DG, Thompson RF. Classical conditioning of the rabbit eyelid response with a mossyfiber stimulation CS: I. Pontine nuclei and middle cerebellar peduncle stimulation. Behav Neurosci 1986; 100: 878-87.

39 Steinmetz JE, Rosen DJ, Woodruff-Pak DS, Lavond DG, Thompson RF. Rapid transfer of training occurs when direct mossy fiber stimulation is used as a conditioned stimulus for classical eyelid conditioning. Neurosci Res 1986; 3: 606-16.

40 Shinkman PG, Swain RA, Thompson RF. Classical conditioning with electrical stimulation of cerebellum as both conditioned and unconditioned stimulus. Behav Neurosci 1996; 110: 914-21.

41 Jirenhed DA, Hesslow G. Time course of classically conditioned Purkinje cell response is determined by initial part of conditioned stimulus. J Neurosci 2011; 31: 9070-4.

42 Poulos AM, Thompson RF. Timing of conditioned responses utilizing electrical stimulation in the region of the interpositus nucleus as a CS. Integr Physiol Behav Sci 2004; 39: 83-94.

43 Jimenez-Diaz L, Navarro-Lopez Jde D, Gruart A, Delgado-Garcia JM. Role of cerebellar interpositus nucleus in the genesis and control of reflex and conditioned eyelid responses. J Neurosci 2004; 24: 913845.

44 Leal-Campanario R, Delgado-Garcia JM, Gruart A. Microstimulation of the somatosensory cortex can substitute for vibrissa stimulation during Pavlovian conditioning. Proc Natl Acad Sci U S A 2006; 103 : 10052-7.

45 Woody CD, Yarowsky PJ. Conditioned eye blink using electrical stimulation of coronal-precruciate cortex as conditional stimulus. J Neurophysiol 1972; 35: 242-52.

46 Troncoso J, Munera A, Delgado-Garcia JM. Classical conditioning of eyelid and mystacial vibrissae responses in conscious mice. Learn Mem 2004; 11: 724-6.

47 Goldman-Rakic PS. Cellular and circuit basis of working memory in prefrontal cortex of nonhuman primates. Prog Brain Res 1990; 85: 325-35; discussion 35-6.
48 Goldman-Rakic PS. Cellular basis of working memory. Neuron 1995; 14: 477-85.

49 Fuster JM. The prefrontal cortex - an update: time is of the essence. Neuron 2001; 30: 319-33.

50 Corbit LH, Balleine BW. The role of prelimbic cortex in instrumental conditioning. Behav Brain Res 2003; 146: 145-57.

51 Powell DA, Skaggs H, Churchwell J, McLaughlin J. Posttraining lesions of the medial prefrontal cortex impair performance of Pavlovian eyeblink conditioning but have no effect on concomitant heart rate changes in rabbits (Oryctolagus cuniculus). Behav Neurosci 2001; 115: 1029-38.

52 Doty RW, Larsen RM, Ruthledge LT Jr. Conditioned reflexes established to electrical stimulation of cat cerebral cortex. J Neurophysiol 1956; 19: 401-15.

53 Simon B, Knuckley B, Churchwell J, Powell DA. Post-training lesions of the medial prefrontal cortex interfere with subsequent performance of trace eyeblink conditioning. J Neurosci 2005; 25: 10740-6.

54 Oswald BB, Maddox SA, Powell DA. Prefrontal control of trace eyeblink conditioning in rabbits: role in retrieval of the CR? Behav Neurosci 2008; 122: 841-8.

55 Kronforst-Collins MA, Disterhoft JF. Lesions of the caudal area of rabbit medial prefrontal cortex impair trace eyeblink conditioning. Neurobiol Learn Mem 1998; 69: 147-62.

56 Chachich M, Powell DA. Both medial prefrontal and amygdala central nucleus lesions abolish heart rate classical conditioning, but only prefrontal lesions impair reversal of eyeblink differential conditioning. Neurosci Lett 1998; 257: 151-4.

57 Powell DA, Skaggs H, Churchwell J, McLaughlin J. Posttraining lesions of the medial prefrontal cortex impair performance of Pavlovian eyeblink conditioning but have no effect on concomitant heart rate changes in rabbits (Oryctolagus cuniculus). Behav Neurosci 2001; 115: 1029-38.

58 Leal-Campanario R, Fairen A, Delgado-Garcia JM, Gruart A. Electrical stimulation of the rostral medial prefrontal cortex in rabbits inhibits the expression of conditioned eyelid responses but not their acquisition. Proc Natl Acad Sci U S A 2007; 104: 11459-64.

59 McLaughlin J, Skaggs H, Churchwell J, Powell DA. Medial prefrontal cortex and pavlovian conditioning: trace versus delay conditioning. Behav Neurosci 2002; 116: 37-47.

60 Rapisarda C, Bacchelli B. The brain of the guinea pig in stereotaxic coordinates. Arch Sci Biol (Bologna) 1977; 61: 1-37.

$61 \mathrm{Martin} \mathrm{JH}$. Autoradiographic estimation of the extent of reversible inactivation produced by microinjection of lidocaine and muscimol in the rat. Neurosci Lett 1991; 127: 160-4.

$62 \mathrm{Martin} \mathrm{JH}$, Ghez C. Pharmacological inactivation in the analysis of the central control of movement. J Neurosci Methods 1999; 86: 145-59.

63 Sarihi A, Motamedi F, Naghdi N, Rashidy-Pour A. Lidocaine reversible inactivation of the median raphe nucleus has no effect on reference memory but enhances working memory versions of the Morris water maze task. Behav Brain Res 2000; 114: 1-9.

$64 \mathrm{Hu} \mathrm{B}$, Chen H, Yang L, Tao ZF, Yan J, Zhang YH, et al. Changes of synaptic ultrastructure in the guinea pig interpositus nuclei associate with response magnitude and timing after trace eyeblink conditioning. Behav Brain Res 2012; 226: 529-37.

65 Kotani S, Kawahara S, Kirino Y. Trace eyeblink conditioning in decerebrate guinea pigs. Eur J Neurosci 2003; 17: 1445-54.

66 Green JT, Arenos JD. Hippocampal and cerebellar single-unit activity during delay and trace eyeblink conditioning in the rat. Neurobiol Learn Mem 2007; 87: 269-84.

67 Vogt $\sqcup$, Vogt BA, Sikes RW. Limbic thalamus in rabbit: architecture, projections to cingulate cortex and distribution of muscarinic acetyl- 
choline, GABAA, and opioid receptors. J Comp Neurol 1992; 319: 205-17.

68 Debiec J, LeDoux JE, Nader K. Cellular and systems reconsolidation in the hippocampus. Neuron 2002; 36: 527-38.

69 Powell DA, Churchwell J. Mediodorsal thalamic lesions impair trace eyeblink conditioning in the rabbit. Learn Mem 2002; 9: 10-7.

$70 \mathrm{Hu} \mathrm{B}$, Chen H, Feng H, Zeng Y, Yang L, Fan ZL, et al. Disrupted topography of the acquired trace-conditioned eyeblink responses in guinea pigs after suppression of cerebellar cortical inhibition to the interpositus nucleus. Brain Res 2010; 1337: 41-55.

71 Garcia KS, Mauk MD. Pharmacological analysis of cerebellar contributions to the timing and expression of conditioned eyelid responses. Neuropharmacology 1998; 37: 471-80.

72 Steinmetz JE. Brain substrates of classical eyeblink conditioning: a highly localized but also distributed system. Behav Brain Res 2000; 110: 13-24.

73 Koutalidis O, Foster A, Weisz DJ. Parallel pathways can conduct visual CS information during classical conditioning of the NM response. J Neurosci 1988; 8: 417-27.

74 Prokasy WF, Kesner RP, Calder LD. Posttrial electrical stimulation of the dorsal hippocampus facilitates acquisition of the nictitating membrane response. Behav Neurosci 1983; 97: 890-6.

75 Kalmbach BE, Ohyama T, Mauk MD. Temporal patterns of inputs to cerebellum necessary and sufficient for trace eyelid conditioning. J Neurophysiol 2010; 104: 627-40.

76 Wada N, Kishimoto Y, Watanabe D, Kano M, Hirano T, Funabiki K, et al. Conditioned eyeblink learning is formed and stored without cerebellar granule cell transmission. Proc Natl Acad Sci U S A 2007; 104: 16690-5.

77 Siegel JJ, Kalmbach B, Chitwood RA, Mauk MD. Persistent activity in a cortical-to-subcortical circuit: bridging the temporal gap in trace eyelid conditioning. J Neurophysiol 2012; 107: 50-64.

78 Okuda Y, Shikata H, Song WJ. A train of electrical pulses applied to the primary auditory cortex evokes a conditioned response in guinea pigs. Neurosci Res 2011; 71: 103-6.

79 Bao S, Chen L, Thompson RF. Learning- and cerebellum-dependent neuronal activity in the lateral pontine nucleus. Behav Neurosci 2000; 114: 254-61.

80 Steinmetz JE, Logan CG, Rosen DJ, Thompson JK, Lavond DG, Thompson RF. Initial localization of the acoustic conditioned stimulus projection system to the cerebellum essential for classical eyelid conditioning. Proc Natl Acad Sci U S A 1987; 84: 3531-5. 